\title{
Acceptability of the Imperial Nutritional Screening System: results of a pilot study
}

\author{
J. D. Tammam, L. Wright and M. Hickson \\ Imperial College Healthcare NHS Trust, Department of Nutrition and Dietetics, Charing Cross Hospital, \\ London W6 8RF, UK
}

Nutritional screening tools are essential in order to identify patients at risk of malnutrition ${ }^{(1)}$, allowing preventative and therapeutic action, which can reduce morbidity and economic burden. Nonetheless, even the best tool is only beneficial if it is used. The ideal screening tool should be quick and easy to use and acceptable to nursing staff ${ }^{(2)}$. The validity and reliability of the Imperial Nutritional Screening System (INSYST) has previously been demonstrated and here the tool's speed and ease of use is described, alongside nurse and patient views on the acceptability of the screening process. INSYST is a two-tiered screening tool that does not require BMI. It comprises a pre-screen (INSYST I; affirmation of recent unintentional weight loss or decreased appetite) and INSYST II (a brief assessment carried out only if triggered by INSYST I that details food intake, weight change and other risk factors; yielding 'not at risk', 'at risk' and 'malnourished' categories).

Twenty nurses gave consent to participate. They were observed and timed carrying out a routine evaluation of INSYST I and II and weighing a new patient. Nurses were asked to rate the tool for speed and ease of use on two four-point Likert scales ${ }^{(3)}$ (1, very slow or difficult; 4, very fast or easy). Nurses and patients were asked to comment on the acceptability of the process.

Most nurses were band 5 (range band 3-band 7) with a median 42 (range 4-156) months service with the Trust and reported using INSYST for a median of 36 (range 5-60) months. INSYST I took a median of 60 (27-135) s to complete, INSYST II 102 (38-250) s and weighing $100(27-191) \mathrm{s}$. The total median time was approximately $5 \mathrm{~min}$ with minimum approximately 1.5 min and maximum approximately $7.5 \mathrm{~min}$. The results from the Likert scales are shown in the Figure.

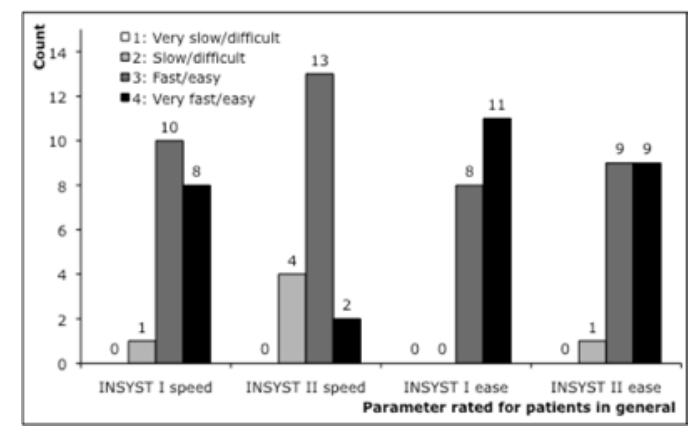

In total $77 \%$ of nurse comments were favourable, focusing on the fact that they felt it was a good tool, it was quick and easy to complete and that it explored relevant variables in identifying malnutrition. Only $23 \%$ of the comments were critical, highlighting the fact that speed and ease depended on the patient, historical weights were often not known and training was needed. All the patients' comments were positive and demonstrated that patients felt the questions asked were relevant and necessary.

The whole INSYST process takes on average $5 \mathrm{~min}$, which is comparable with other screening tools ${ }^{(3)}$. The two-tier system means that many patients will not need INSYST II and thus the process will only take on average $2.5 \mathrm{~min}$. This flexibility is reflected in the nurses grading the tool as fast and easy to use, and is further supported by their comments. Speed and ease of use is an important consideration for nurses, given the pressures and time constraints they face. If the tool does not meet these basic criteria, there is a risk that nutritional screening is not completed, with serious consequences for patients left without appropriate nutritional support. The patient comments indicate that patients appreciate the importance of being nutritionally screened and are happy that this process takes place. This approval is important as patient refusal to complete routine screening would negate the utility of any screening tool. INSYST is quick and easy to use and acceptable to both nurses and patients. By meeting this basic requirement, INSYST is a viable tool to introduce for routine nutritional screening. As this study was a relatively small pilot study, further research is warranted.

1. National Institute for Health and Clinical Excellence (2006) Nutrition Support in Adults: Oral Nutrition Support, Enteral Tube Feeding and Parenteral Nutrition. Clinical Guideline 32. London: NICE.

2. Green SM \& Watson R (2006) J Adv Nurs 54, 477-490.

3. Stratton RJ, Hackston A, Longmore D et al. (2004) Br J Nutr 92, 799-808. 